\title{
Assessment of non-disabled individuals walking with partial body weight support on a treadmill and over the ground
}

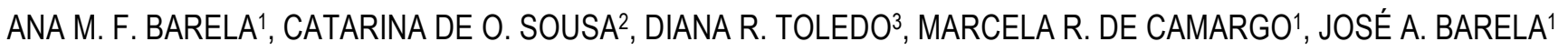 \\ ${ }^{1}$ Cruzeiro do Sul University | ${ }^{2}$ Federal University of Rio Grande do Norte - Department of Physical Therapy | 3University of São Paulo - Biomedical Engineering Laboratory \\ Correspondence to: Ana Maria Forli Barela, Universidade Cruzeiro do Sul, Instituto de Ciências da Atividade Física e Esporte, Rua Galvão Bueno, 868, 13०. andar, Bloco \\ B, São Paulo, SP, Brasil 01506-000
}

Email: ana.barela@cruzeirodosul.edu.br; ambarela@gmail.com

\begin{abstract}
AT A GLANCE
Gait pattern of healthy adults is different when performed over the ground compared to treadmill; BWS employed over the ground provides a more stable walking pattern of healthy adults; Walking surface should be considered before employing BWS.
\end{abstract}

\section{ACKNOWLEDGEMENTS}

Ana M. F. Barela is thankful to CNPq for Postdoc fellowship (\#151896/2006-2).

Marcela R. de Camargo is thankful to FAPESP for Doctoral fellowship (\#2012/14634-9).

\begin{abstract}
BACKGROUND: Partial body weight support (BWS) systems have been used as a strategy for rehabilitation of individuals with gait impairment. More recently, some studies have described BWS over the ground, which is the daily life-walking surface. However, before implementing rehabilitation protocols for individual with gait impairments, it is important to investigate some walking parameters in healthy individuals walking with BWS on both treadmill and over the ground.

AIM: To describe gait kinematic parameters of healthy adults by manipulating BWS and two different ground surfaces.

METHOD: Kinematics data from 14 healthy adults were acquired while they walked wearing a harness with $30 \%$ BWS and with no harness on treadmill and over the ground. Temporal-spatial walking parameters and joint and segmental angles were calculated.

RESULTS: Several walking parameters were different between walking surfaces, and BWS influenced these parameters according to walking surface. In general, participants walked faster and with longer strides and larger body segments excursion over the ground compared to the treadmill. Also, participants presented the most stable walking pattern as they walked over the ground with BWS.

CONCLUSION: Changes presented by healthy adults were towards an adjustable and adaptable pattern performed over the ground that provided a more stable surface.
\end{abstract}

\section{INTRODUCTION}

Partial body weight support (BWS) systems have been used as a strategy for therapeutic gait training of individuals with neurological impairments ${ }^{1-3}$. Most of BWS systems consist of a mounting frame and harness to support a percentage of the individual's body weight as they walk on a motorized treadmill. The rationale for using a BWS system is that the reduction of gravitational forces would reduce the load that should be overcome by the individual, facilitating the walking requirements. Consequently, this strategy might promote a gait pattern close to normal. ${ }^{3}$

Usually, the percentage of BWS reduction ranges from $10 \%$ to $70 \% .{ }^{4}$ However, Threlkeld et al. ${ }^{5}$ verified that kinematics, temporal, and spatial gait characteristics of young healthy adults had minimum variation at $10 \%$ and $30 \%$ BWS, and became different at $50 \%$ and $70 \%$ BWS. Among all these different percentage levels of BWS, $30 \%$ is the most used for individuals with stroke, for example, as it yields to gait pattern improvements. ${ }^{6,7}$

Besides the appropriate percentage of body weight unloading during walking on treadmill, any intervention protocol should consider appropriate conditions such as those close to daily life activities in order to promote and maximize skills transfer. ${ }^{8}$ Unfortunately,

\begin{tabular}{l|l|l|l|l|}
\hline $\begin{array}{l}\text { Barela, Sousa, } \\
\text { Toledo, Camargo \& } \\
\text { Barela }\end{array}$ & 2015 & VOL. 9 & N.1 & 1 of 10 \\
& & &
\end{tabular}


the strategies adopted to walk on treadmills are different from the ones adopted to walk over ground in terms of propulsion and balance control ${ }^{9}$ and might jeopardize a direct skill transfer from intervention to daily walking conditions. Moreover, the speed adopted to walk on treadmills is not self-selected as it can be when one walks over ground.10-14 These factors might influence gait characteristics, such as kinematics, temporal-spatial, ${ }^{10}$ kinetics, ${ }^{14}$, and electromyography ${ }^{12}$ during the gait cycle.

Although these differences had been examined in healthy adults ${ }^{10,12,13}$ and individuals with stroke, ${ }^{11,14}$ only few studies had compared walking with BWS system on both treadmill and over ground, where the participants were children with cerebral palsy, 15,16, and individuals with stroke. ${ }^{17}$ Previous study had investigated individuals with stroke walking over the ground with BWS, ${ }^{18}$ and exposed them to over ground gait training with BWS. ${ }^{19}$ Therefore, walking with BWS over the ground might constitute an alternative and important gait training protocol for individuals with gait impairments. However, in order to implement and decide about walking rehabilitation protocols using BWS, it is necessary to uncover several issues related to the influences that such a system, on both surfaces, could promote in non-disabled individuals walking pattern. Thus, the goal of this study was to describe gait kinematic parameters of healthy young adults by manipulating BWS and two different surfaces. We hypothesized that walking with BWS over the ground would be more related to normal walking because its requirements are closer to daily life activities compared to walking on treadmill.

\section{METHODS}

\section{Participants}

Fourteen healthy young adults (8 males, 6 females) participated in this study. Mean ( \pm SD) age, height, and body mass were $26( \pm 3)$ years, $1.71( \pm 0.1) \mathrm{m}$, and $69( \pm$ 16) $\mathrm{kg}$, respectively. In order to take part of the study, participants should not have any known gait impairment, be aged between 20 and 30 year-old, and not have previous experience with the BWS apparatus used in this study. All participants signed an informed consent agreement, as approved by the ethics committee of Institute of Biosciences, São Paulo State University.

\section{Instrumentations, tasks and procedures}

Figure 1 (A) illustrates a partial view of the BWS system employed in this study. This system consists of a harness with adjustable belts and padded straps for the thighs, similar to the one used by Norman et al. ${ }^{9}$, which was attached to a horizontal bar. A steel cable from an electric motor pulled the horizontal bar upward and slid it through an upper rail as an individual walks. A load cell connects the horizontal bar to the cable and measures the amount of weight borne by the BWS system, which was shown on a digital display. In order to support the weight, individual stays still until the motor is activated by the experimenter, who lengthened or shortened the cable to bear the desired amount of body weight. During the trials using the BWS system, participants were mechanically supported by the system.

Infrared Emitting Diodes (IRED) markers were attached on an adapted support (Figure 1-B) and this support was placed on the following locations of participants' body in order to acquire kinematics data: right and left head of fifth metatarsal, right lateral 
malleolus, right head of fibula, right lateral epicondyle of the femur, right greater trochanter, and right acromion (Figure 1-C), and data were registered using the OPTOTRAK system (Northern Digital, Inc., Waterloo, Canada), displaced behind the participant (Figure 1-A), at a frequency of $100 \mathrm{~Hz}$.

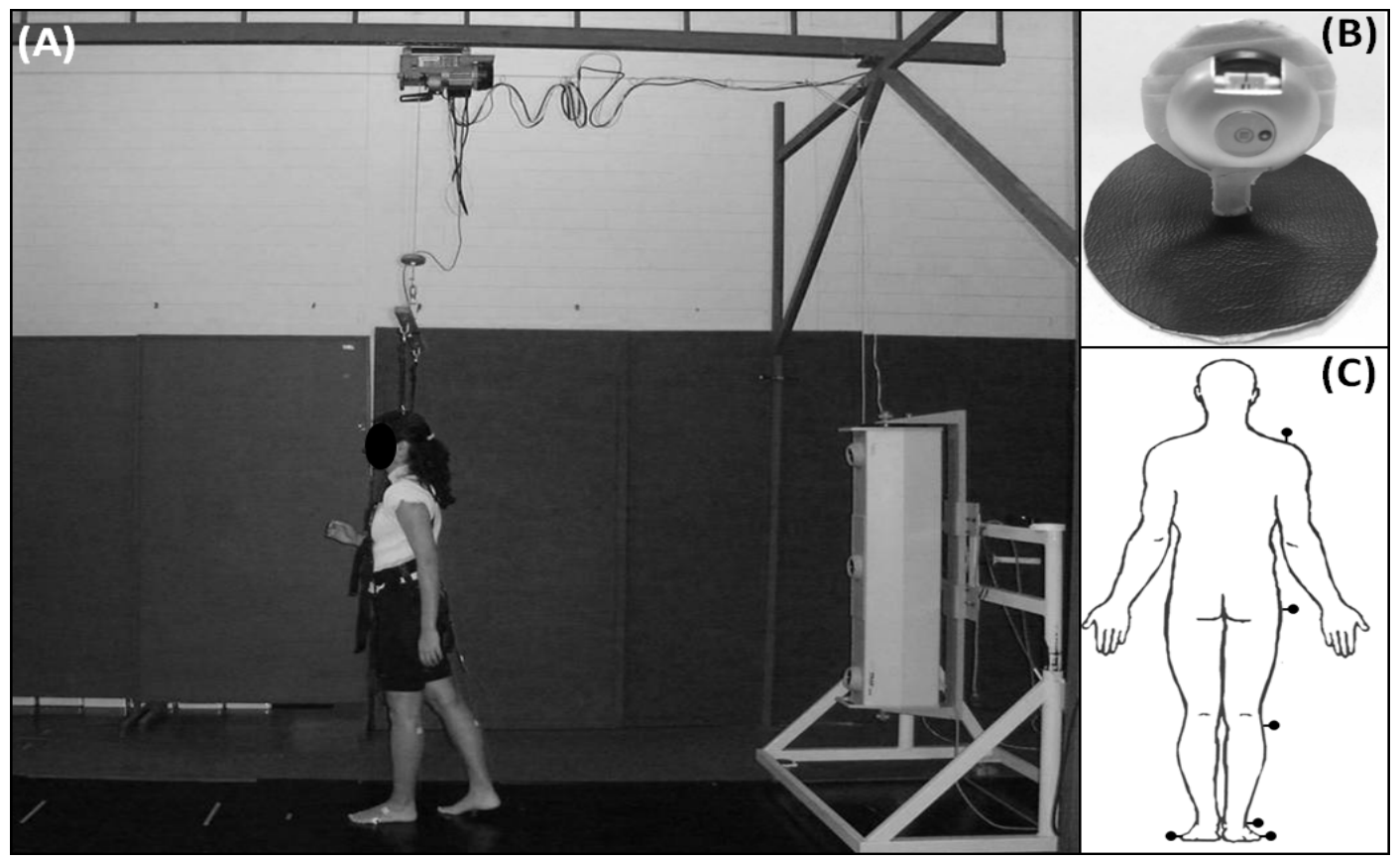

Figure 1. Partial view of the BWS system employed in this study for the over ground condition and the OPTOTRAK block camera setup to acquire the kinematics data (A); illustration of the device used to fixate the IREDs facing backwards (B); and the representation of IREDs fixation on the participant as viewed from the OPTOTRAK block camera position (C).

Participants were assessed in four conditions, in the following same order: (1) walking over the ground wearing a harness with $30 \%$ BWS (in an attempt to maintain the similar walking speed among the conditions and assuming that participants would walk slower with body unloading over the ground); (2) walking over the ground with no harness (for practical reasons and to minimize the time spent by the participant in the laboratory, we grouped first and second conditions over the ground); (3) on a treadmill with no harness (to minimize any IRED marker position on the participants' body due the harness manipulation, i.e., wearing and removing it among experimental conditions); and (4) on a treadmill wearing a harness with $30 \%$ BWS. For the conditions that participants had to walk on a treadmill, a motorized treadmill (Athletic Advanced 2) was placed in the middle of the walkway.

Prior to the experimental procedures, participants were adjusted to the $30 \%$ BWS and requested to walk as comfortable as possible. The obtained speed by each participant was required to be similar in all experimental conditions. For the over ground conditions, participants performed five trials with and without harness. For the treadmill conditions, participants walked for five minutes, with no harness and for five minutes with harness, before any data acquisition. After that, data were acquired through five periods of five seconds each, without the participants' awareness. In addition, data for a three-second 
period were acquired, in which participants stood still upright on the center of the walkway and on center of the treadmill before each different experimental condition, for reference purpose.

\section{Data Analysis}

Three-dimensional coordinates were obtained from each IRED marker and used to calculate spatial-temporal and angular parameters. A gait stride per trial was determined from two consecutive right foot contacts, and it was analyzed for each participant in each experimental condition. Data analyses were performed using specific Matlab routines (Mathworks, Inc.). First, data were digitally filtered using a $4^{\text {th }}$ order and zero-lag Butterworth filter and all markers were low-pass filtered at $10 \mathrm{~Hz}$. For joint and segmental angles, strides were normalized in time from $0 \%$ to $100 \%$, with a $1 \%$ step. These cycles were referenced to the participants' neutral angles measured during the reference trial in each condition and were then averaged to obtain the mean cycle for each participant. The same procedure was repeated to obtain the mean cycle among participants.

The following data were calculated: mean walking speed, stride length and speed, cadence, first and second double support, single support, and swing duration, ankle, knee, and hip joints range of motion (ROM), and foot, shank, thigh, and trunk segmental ROM, in the sagittal plane. The movements of the segments were counter-clockwise (backward) and clockwise (forward) rotations around the medial-lateral axis on the sagittal plane, which denoted positive and negative values, respectively. For example, a counterclockwise rotation of the trunk means trunk extension from neutral position and a clockwise rotation means trunk flexion from neutral position.

Data from three repetitions under each condition were averaged for each participant. Statistical analyses involved an univariate analysis of variance (ANOVA) and four multivariate analyses of variance (MANOVAs) for repeated measures, having as factor the four conditions (over ground walking with 30\% BWS, over ground walking with no harness, treadmill walking with $30 \%$ BWS, and treadmill walking with no harness). The dependent variables were the mean walking speed for the ANOVA; stride length, stride speed and cadence for the first MANOVA; first double support, single support, second double support, and swing durations for the second MANOVA; ankle, knee, and hip joint ROM for the third MANOVA; and foot, shank, thigh, and trunk ROM for the fourth MANOVA. Post hoc tests with Bonferroni adjustments were employed to the pairwise comparisons when necessary. An alpha level of 0.05 was used for all statistical tests, which were performed using the SPSS software (SPSS Inc.).

\section{RESULTS}

Table 1 depicts mean ( \pm SD) values of the investigated variables and the univariate statistical results. Although requested to maintain similar walking speed for all conditions, the participants presented higher speed when they walked over the ground with no harness compared to the remained conditions. Stride length and speed were longer and higher, respectively, when they walked over the ground compared to treadmill, and cadence was higher when they walked over the ground with no harness than when they walked on treadmill (Table 1). 
Regarding stance and swing phase duration, the first and second double support durations were shorter when the participants walked over the ground with $30 \%$ BWS compared to the remained conditions, and it was shorter when they walked either over the ground with no harness or on treadmill with $30 \%$ BWS compared to treadmill with no harness. On the other hand, the single support and the swing durations were longer when the participants walked over the ground with 30\% BWS compared to the remained conditions, and it was longer when they walked either over the ground with no harness or on treadmill with $30 \%$ BWS compared to treadmill with no harness (Table 1).

Table 1: Mean $( \pm S D)$ values of temporal-spatial parameters and joint and segmental range of motion (ROM) during walking with $30 \%$ BWS and with no harness over ground and on treadmill $(n=14)$.

\begin{tabular}{|c|c|c|c|c|c|c|}
\hline \multirow{4}{*}{ Variables } & \multicolumn{6}{|l|}{ Conditions } \\
\hline & \multicolumn{2}{|l|}{ Overground } & \multicolumn{2}{|l|}{ Treadmill } & \multirow{3}{*}{$F$ ratio } & \multirow{3}{*}{$p$ value } \\
\hline & $30 \%$ BWS & No harness & $30 \%$ BWS & No harness & & \\
\hline & Mean (SD) & Mean (SD) & Mean (SD) & Mean (SD) & & \\
\hline Mean walking speed $(\mathrm{m} / \mathrm{s})$ & $0.94(0.14)^{a}$ & $0.99(0.12)^{a, b, c}$ & $0.88(0.16)^{b}$ & $0.88(0.16)^{c}$ & 9.75 & 0.003 \\
\hline Stride length $(\mathrm{m})$ & $1.15(0.13)^{a, b}$ & $1.17(0.12)^{\mathrm{c}, \mathrm{d}}$ & $0.72(0.19)^{\mathrm{a}, \mathrm{c}}$ & $0.72(0.18)^{\mathrm{b}, \mathrm{d}}$ & 187 & $<0.001$ \\
\hline Stride speed $(\mathrm{m} / \mathrm{s})$ & $0.95(0.15)^{a, b}$ & $1.01(0.13)^{\mathrm{c}, \mathrm{d}}$ & $0.59(0.20)^{\mathrm{a}, \mathrm{c}}$ & $0.59(0.20)^{b, d}$ & 180 & $<0.001$ \\
\hline Cadence (steps/min) & $99(8.40)$ & $103(5.25)^{a, b}$ & $96(8.78)^{a}$ & $96(8.99)^{b}$ & 11 & $<0.001$ \\
\hline $1^{\text {st }}$ double support (\%) & $9.93(2.09)^{a, b, c}$ & $12.27(1.40)^{\mathrm{a}, \mathrm{d}}$ & $12.50(2.05)^{\mathrm{b}, \mathrm{e}}$ & $14.57(2.28)^{\mathrm{c}, \mathrm{d}, \mathrm{e}}$ & 27.32 & $<0.001$ \\
\hline Single stance $(\%)$ & $40.44(2.12)^{a, b, c}$ & $37.97(1.65)^{\mathrm{a}, \mathrm{d}}$ & $37.74(2.49)^{\mathrm{b}, \mathrm{e}}$ & $35.24(2.44)^{c, d, e}$ & 34.29 & $<0.001$ \\
\hline $2^{\text {nd }}$ double support (\%) & $9.63(2.32)^{a, b, c}$ & $12.30(1.61)^{\mathrm{a}, \mathrm{d}}$ & $12.31(2.32)^{\mathrm{b}, \mathrm{e}}$ & $14.23(1.85)^{c, d, e}$ & 29.11 & $<0.001$ \\
\hline Swing $(\%)$ & $40.00(1.95)^{a, b, b}$ & $37.46(1.29)^{\mathrm{a}, \mathrm{d}}$ & $37.45(1.79)^{\mathrm{b}, \mathrm{e}}$ & $35.96(1.73)^{c, d, e}$ & 37.98 & $<0.001$ \\
\hline \multicolumn{7}{|l|}{ Joint ROM ( $\left.{ }^{\circ}\right)$} \\
\hline Ankle & $30.3(5.3)^{a, b}$ & $27.9(7.0)^{\mathrm{c}, \mathrm{d}}$ & $20.9(4.2)^{\mathrm{a}, \mathrm{c}}$ & $21.4(3.2)^{\mathrm{b}, \mathrm{d}}$ & 17.19 & $<0.001$ \\
\hline Knee & $60.4(5.7)^{\mathrm{a}, \mathrm{b}, \mathrm{c}}$ & $63.5(6.4)^{\mathrm{a}, \mathrm{d}, \mathrm{e}}$ & $57.1(6.1)^{b, d}$ & $57.6(7.0)^{c, e}$ & 20.08 & $<0.001$ \\
\hline Hip & $28.3(4.1)$ & $29.4(4.3)$ & $27.8(4.5)$ & $27.97(4.4)$ & 1.67 & 0.201 \\
\hline \multicolumn{7}{|l|}{ Segmental ROM $\left({ }^{\circ}\right)$} \\
\hline Foot & $82.5(7.6)^{a, b}$ & $84.8(9.0)^{\mathrm{c}, \mathrm{d}}$ & $70.1(9.4)^{\mathrm{a}, \mathrm{c}}$ & $70.7(10.3)^{\mathrm{b}, \mathrm{d}}$ & 43.91 & $<0.001$ \\
\hline Shank & $68.4(4.8)^{a, b, c, c}$ & $72.7(5.3)^{\mathrm{a}, \mathrm{d}, \mathrm{e}}$ & $62.0(5.9)^{b, d, f}$ & $64.1(7.4)^{c, e, f f}$ & 52.49 & $<0.001$ \\
\hline Thigh & $33.0(4.0)$ & $35.7(3.9)^{\mathrm{a}, \mathrm{b}}$ & $31.8(4.8)^{\mathrm{a}}$ & $32.8(4.3)^{\mathrm{b}}$ & 9.58 & $<0.001$ \\
\hline Trunk & $7.2(1.4)^{\mathrm{a}}$ & $9.1(1.3)^{a, b, b}$ & $6.1(1.6)^{\mathrm{b}, \mathrm{d}}$ & $8.0(1.4)^{\mathrm{c}, \mathrm{d}}$ & 15.76 & $<0.001$ \\
\hline
\end{tabular}

Figure 2 depicts the mean $( \pm S D)$ stride cycle profile of ankle, knee and hip joints of all participants walking over the ground (Figure 2-A) and on the treadmill (Figure 2-B) with $30 \%$ BWS and with no harness. Similar patterns were observed when respective joints were compared among the four conditions, although either more flexed or more extended excursion of the joint can be observed. Univariate tests revealed differences for most of joint ROM (Table 1). For instance, ankle joint presented more excursions when 
participants walked over the ground compared to treadmill. Similar results occurred for the knee, although this joint presented larger excursion when participants walked over the ground with no harness compared to $30 \%$ BWS. Finally, no difference was found for the hip joint among all conditions.
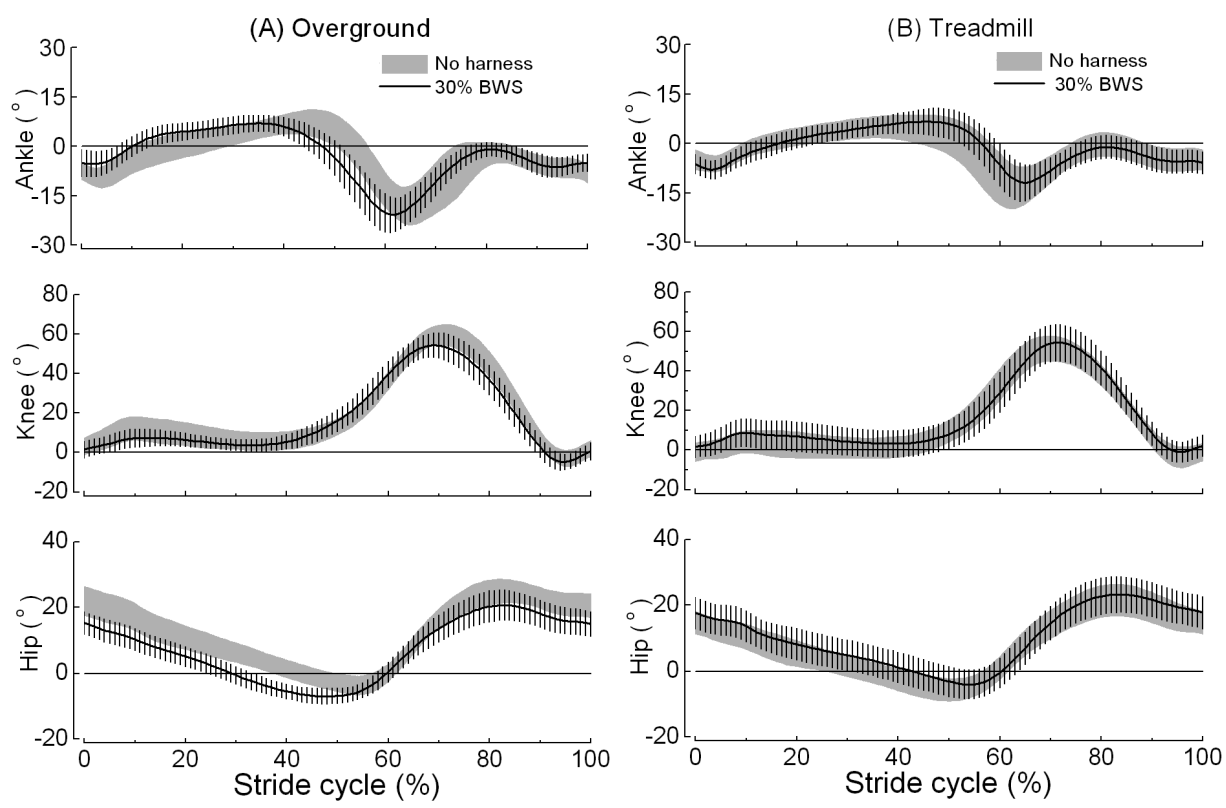

Figure 2. Mean ( $\pm \mathrm{SD}$ ) stride cycle of ankle, knee, and hip joint angles of the participants walking with no harness (grey area) and with 30\% BWS (line) overground (A) and on treadmill (B). Note: positive values denote ankle dorsiflexion, knee and hip flexion, and negative values denote ankle plantar flexion, knee and hip extension $(n=14)$.

Figure 3 depicts the mean $( \pm S D$ ) stride cycle profile of foot, shank, thigh, and trunk segments of all participants walking over the ground (Figure 3-A) and on treadmill (Figure 3B) with 30\% BWS and with no harness. Similar patterns were observed when respective segments were compared among all conditions.

Univariate tests indicated difference for all segments ROM (Table 1). The foot presented larger excursion when participants walked over the ground compared to treadmill and the shank presented the largest excursion when they walked over the ground with no harness, and the smallest excursion when they walked on treadmill with $30 \%$ BWS. Yet, the shank presented larger excursion when participants walked over the ground with $30 \%$ BWS compared to treadmill. On the other hand, the thigh presented larger excursion when participants walked over the ground with no harness compared to treadmill. Finally, the trunk presented larger excursion when participants walked over the ground with no harness compared to the remained conditions and when they walked on treadmill with no harness compared to treadmill with $30 \%$ BWS. 

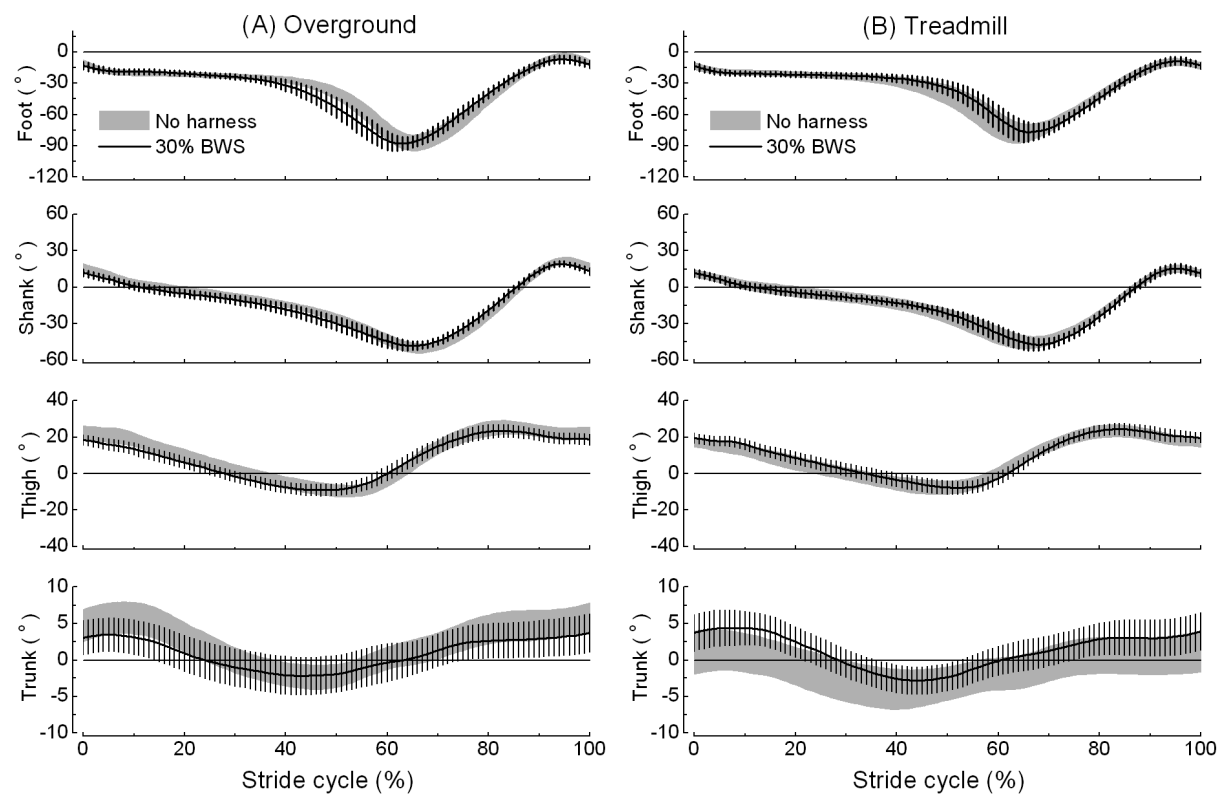

Figure 3. Mean $( \pm S D)$ stride cycle of foot, shank, thigh, and trunk of the participants walking with no harness (grey area) and with 30\% BWS (line) overground (A) and on treadmill (B). Note: positive values denote counter-clockwise (backward) rotation of the segments and negative values denote clockwise (forward) rotation of the segments $(n=14)$.

\section{DISCUSSION}

This study examined healthy young adults walking with BWS system over the ground and on a treadmill. The results revealed that walking over the ground is different of walking on a treadmill, and the effects of partial BWS are also dependent of the surface in which body weight unloading is provided. In general, these individuals presented longer and faster strides when they walked over the ground compared to treadmill, and the BWS system allowed them to present a more stable walking pattern over the ground than on treadmill. In addition, walking over the ground with and without the harness was characterized by larger ankle and knee range of motion.

The over ground surface allowed a walking pattern with longer and faster strides than on treadmill and, most likely, these differences between surfaces could be due to the length of the treadmill, which might influence step length ${ }^{20}$ and, consequently, stride speed. If this were the case, overground constitutes a much more suitable condition for gait training and rehabilitation mainly because it would allow more opportunities and flexibility for patients to adjust walking parameters according to their needs.

Another important issue regarding gait training and rehabilitation is the stability of gait pattern. In this case, walking with BWS over the ground provided the most stable condition for healthy young adults as they presented shorter double support and longer single support durations compared to treadmill walking. It is well-established that single stance duration indicates the capability of limb support ${ }^{21}$ as well as the decrease of double support duration indicates walking stability. In this way, 30\% BWS over the ground provided the most stable condition even for healthy young adults. Conversely, treadmill does not provide a stable surface for walking even with BWS. Our results revealed that double and single stance durations of healthy adults walking on treadmill with BWS were 
similar to when they walked over ground with no harness only. These results should be taken into account in the process of mobility reestablishment, as individuals with gait impairment are usually struggling to maintain the upright position and, therefore, they would take even more advantages walking partially supported by the BWS over the ground.

Although our findings are not in agreement with previous study with healthy young adults walking on treadmill with BWS, ${ }^{3}$ the differences are probably due to experimental procedures. Finch et al. ${ }^{3}$ decreased the treadmill speed as the percentage of BWS increased and attributed the differences not to the speed but to the percentage of body unloading. Despite of these divergent results, walking on a treadmill is a challenging task for anyone and it might be even more challenging for individuals with gait impairment, mostly because the moveable supportive surface challenges the stability of the upright position. On the other hand, walking with BWS over the ground would provide means for gait improvements without threatening a very important issue such as the upright stability.

The joint angle profiles presented in this study are similar to previous studies that investigated healthy individuals walking on treadmill and over the ground with no BWS system, ${ }^{20,22}$ and walking on treadmill with BWS. 3,5 Our results, however, indicate that the over ground surface allows larger range of motion of the lower limb joints of healthy young adults either with or without body weight unloading compared to treadmill. The excursion of ankle and knee joints are critical for progression, shock absorption, and limb advancement throughout stride cycle ${ }^{21,23}$ and, therefore, walking over the ground might allow walkers to take advantage of such larger joint motion. These aspects are crucial for gait training and rehabilitation and the over ground surface could be suitable for achieving such goals in many cases of gait impairment. Finally, the lack of difference on the range of motion of hip joint among the conditions tested in this study indicates that even though the harness the participants wore contacted directly thighs, pelvis and trunk segments, the BWS system employed in this study did not constraint the excursion of hip joint throughout stride cycle.

The BWS system employed in this study seems to provide a better body alignment during walking. In this case, the trunk is a critical segment, which has its alignment related to functional performance. ${ }^{24}$ Our results indicate that this system not only reduced the excursion of trunk, but also aligned it closer to the vertical orientation (Figure 3) even in healthy young adults. Such a different orientation might also be related to some walking parameter changes, such as spatial-temporal and range of motions, since it provides a complete re-orientation of the body in space and would be probably more dramatic in individuals with gait impairment, whose segmental orientation are usually altered. This issue should be a critical aspect for those that plan to implement protocols for gait intervention using BWS system.

\section{CONCLUSION}

This study was intended to assess a very feasible BWS system that can be employed either over the ground or on treadmills, and it presented some limitations. Only healthy adults and only $30 \%$ BWS were tested on both surfaces. Healthy participants were chosen because it is important to examine possible effects of the BWS on able-bodied individuals, and to uncover its effects and impact that might have on individuals with gait impairment. The $30 \%$ of body unloading was chosen because it has been the most employed percentage of body unloading intervention protocols. ${ }^{6-7}$ In addition, the order of 
the experimental conditions was not randomized mainly because of practical reasons and since we planned to have each participant adopting a walking speed that should be maintained in all experimental conditions without changes in movement pattern, as described earlier in methods. However, this was not the case since slight higher speed was observed to the non-harness condition compared to the other conditions. These limitations have been addressed in following studies in which different percentages of body weight unloading and walking speed have been investigated employing the BWS system on both surfaces. Finally, different analyses are underway besides kinematics to have a full understanding of gait under the different experimental conditions.

\section{REFERENCES}

1. Schindl MR, Forstner C, Kern H, Hesse S. Treadmill training with partial body weight support in nonambulatory patients with cerebral palsy. Arch Phys Med Rehabil 2000; 81(3): 301-6.

2. Sullivan KJ, Knowlton BJ, Dobkin BH. Step training with body weight support: effect of treadmill speed and practice paradigms on poststroke locomotor recovery. Arch Phys Med Rehabil 2002; 83(5): 683-91.

3. Finch L, Barbeau H, Arsenault B. Influence of body weight support on normal human gait: development of a gait retraining strategy. Phys Ther 1991; 71(11): 842-56.

4. Miyai I, Fujimoto $Y$, Ueda $Y$, Yamamoto H, Nozaki S, Saito T, et al. Treadmill training with body weight support: its effect on Parkinson's disease. Arch Phys Med Rehabil 2000; 81(7): 849-52.

5. Therlkeld AJ, Cooper LD, Monger BP, Craven AN, Haupt HG. Temporospatial and kinematic gait alterations during treadmill walking with body weight suspension. Gait Posture 2003; 17(3): 235-45.

6. Lamontagne A, Fung J. Faster is better: implications for speed-intensive gait training after stroke. Stroke 2004; 35: 2543-8.

7. Visintin M, Barbeau H, Korner-Bitensky N, Mayo NE. A new approach to retrain gait in stroke patients through body weight support and treadmill stimulation. Stroke 1998; 29: 1122-8.

8. Richards CL, Malouin F, Wood-Dauphinee S, Williams JI, Bouchard JP, Brunet D. Taskspecific physical therapy for optimization of gait recovery in acute stroke patients. Arch Phys Med Rehabil 1993; 74(6): 612-20.

9. Norman KE, Pepin A, Ladouceur M, Barbeau H. A treadmill apparatus and harness support for evaluation and rehabilitation of gait. Arch Phys Med Rehabil 1995; 76: 772-8.

10. Alton F, Baldey L, Caplan S, Morrisey MC. A kinematic comparison of overground and treadmill walking. Clin Biomech 1998; 13: 434-40.

11. Bayat R, Barbeau H, Lamontagne A. Speed and temporal-distance adaptations during treadmill and overground walking following stroke. Neurorehabil Neural Repair 2005; 19(2): 115-24.

12. Harris-Love ML, Macko RF, Whitall J, Forrester LW. Improved hemiparetic muscle activation in treadmill versus overground walking. Neurorehabil Neural Repair 2004; 18(3): 154-60. 
Brazilian Journal of Motor Behavior

13. Lee SJ, Hidler J. Biomechanics of overground versus treadmill walking in healthy individuals. J Appl Physiol 2008; 104(3): 747-55.

14. Warabi T, Kato M, Kiriyama K, Yoshida T, Kobayashi N. Treadmill walking and overground walking of human subjects compared by recording sole-floor reaction force. Neurosci Res 2005; 53(3): 343-8.

15. Matsuno VM, Camargo MR, Palma GC, Alveno D, Barela AM. Analysis of partial body weight support during treadmill and overground walking of children with cerebral palsy. Rev Bras Fisioter 2010; 14(5): 404-10.

16. Celestino ML. Efeitos da manipulação do tipo de superfície e alívio de peso corporal durante 0 andar de crianças com paralisia cerebral [Dissertação]: Universidade Cruzeiro do Sul; 2014.

17. Segura MSP. O andar de pacientes hemiplégicos no solo e na esteira com suporte total e parcial de peso [Dissertação de Mestrado]. Rio Claro: Universidade Estadual Paulista; 2005.

18. Sousa CO, Barela JA, Prado-Medeiros CL, Salvini TF, Barela AMF. The use of body weight support on ground level: an alternative strategy for gait training of individuals with stroke. J Neuroeng Rehabil 2009; 6(1): 43.

19. Sousa CO, Barela JA, Prado-Medeiros CL, Salvini TF, Barela AM. Gait training with partial body weight support during overground walking for individuals with chronic stroke: a pilot study. J Neuroeng Rehabil 2011; 8: 48.

20. Murray MP, Spurr GB, Sepic SB, Gardner GM, Mollinger LA. Treadmill vs. floor walking: kinematics, electromyogram, and heart rate. J Appl Physiol 1985; 59(1): 87-91.

21. Perry J. Gait analysis Throfare: Slack; 1992.

22. Riley PO, Paolini G, Della Croce U, Paylo KW, Kerrigan DC. A kinematic and kinetic comparison of overground and treadmill walking in healthy subjects. Gait Posture 2007; 26(1): 17-24.

23. Inman VT, Ralston HJ, Todd F. Human locomotion. In: Rose J, Gamble JG, editors. Human walking. 2nd ed. Baltimore: Williams \& Wilkins; 1994. p. 1-22.

24. Hirose D, Ishida K, Nagano $Y$, Takahashi T, Yamamoto H. Posture of the trunk in the sagittal plane is associated with gait in community-dwelling elderly population. Clin Biomech 2004; 19(1): 57-63.

Citation: Barela AMF, Sousa CO, Toledo DR, Camargo MR, Barela JA Assessment of non-disabled individuals walking with partial body weight support on a treadmill and over the ground. BJMB. 2015: 9(1): 1-10.

Editor: Joao A. C. Barros, California State University Fullerton, Fullerton, CA, USA.

Copyright: @ 2015 Barela, Sousa, Toledo, Camargo, Barela and BJMB. This is an open-access article distributed under the terms of the Creative Commons Attribution-NonCommercial-NoDerivatives 4.0 International License which permits unrestricted use, distribution, and reproduction in any medium, provided the original author and source are credited.

Competing interests: The authors have declared that no competing interests exist.

Download: http://socibracom.com/bjmb/index.php/bjmb/issue/view/24 